\title{
A Unified Representation of Stress-Strain Curves in Reversed Direction of Prestrained Cell-Forming Metals
}

\author{
By Tadashi Hasegawa*, Takao Yakou* and U. F. Kocks**
}

\begin{abstract}
A model of the Bauschinger effect for cell-forming metals is summarized, in which a strain in the reversed direction is caused by "unpiling" of dislocations dynamically piled up in the cell interior during prestraining. The model suggests that, if both stress, $\sigma$, and strain, $\varepsilon_{\mathrm{r}}$, in the reversed direction are normalized by the prestress, $\sigma_{\mathrm{p}}, \sigma / \sigma_{\mathrm{p}}$ vs $\varepsilon_{\mathrm{r}} / \sigma_{\mathrm{p}}$ curves become coincident with one another.

Except after prestraining to small strains $(<\sim 3 \%)$, the normalization by $\sigma_{\mathrm{p}}$ was found to be applicable to changes in prestrain and grain size in room-temperature deformation of aluminium and copper, the normalized curves for different prestrains and grain sizes becoming similar in shape. This indicates that the present model can explain the Bauschinger effect so far as the cell structure forms during prestraining. However, the normalization by $\sigma_{\mathrm{p}} / \mu$ ( $\mu$ : the shear modulus) failed for changes in deformation temperature and stacking-fault energy, namely for a change in the nature of dislocation structure (that is, for the conversion of cell structure to subgrain structure or to planar arrays of dislocations). For presenting a more widely applicable model, a quantitative change in a ratio of the number of dynamically stored dislocations during prestraining to that of total dislocations (namely, a fraction of reversely mobile dislocations) with dislocation structure must be taken into consideration.
\end{abstract}

(Received December 23, 1985)

Keywords: stress reversal, Bauschinger effect, dislocations, cell structure, aluminium, copper

\section{Introduction}

In metals which have been plastically deformed in one direction, the yield and flow stresses during unloading and reversed loading are lower than those in the same direction as that of prestraining. This phenomenon is widely known as the Bauschinger effect ${ }^{(1)}$. The degree of the effect has been estimated by several parameters, such as the drop in the yield stress ${ }^{(2)}$ or the proof stress ${ }^{(3)}$ at stress reversal, the strain in the reversed direction (the Bauschinger strain) $)^{(4)(5)}$ and the energy which may help the deformation at the time of reversed loading ${ }^{(6)}$. However, it was found in comparison between zinc and an aluminium-4 mass $\%$ copper alloy containing $\theta^{\prime}$ or $\theta$ precipitates that the deviation from the elastic behaviour at a higher stress during unloading does not necessarily lead to a larger

* Department of Mechanical Engineering, Tokyo University of Agriculture and Technology, Koganeishi, Tokyo 184, Japan.

** Center for Materials Science, Los Alamos National Laboratory, Los Alamos, NM 87545, USA.
Bauschinger strain ${ }^{(6)}$. Further, when different parameters obtained at an earlier or later stage of reversed deformation were used, different conclusions were drawn on temperature- ${ }^{(7)}$ and orientation ${ }^{(8)}$ dependences of the effect. These facts suggest that, for a complete understanding of the effect, the whole shape of the stressstrain curve in the reversed direction (the Bauschinger curve) must be taken into consideration.

To the authors' knowledge, no generally accepted model for the Bauschinger effect, on the basis of which one can explain the whole shape of the Bauschinger curve, has been presented hitherto. The effect is known at present to be mainly determined by dislocation configurations within grains developed during prestraining, not by the existence of grain boundaries ${ }^{(9)}$ ${ }^{(10)}$. Further, the most common dislocation configuration in fcc metals developed during room-temperature deformation is the cell structure. In the present work, a model for the effect is first summarized on the basis of the formation of cell structure. Then, the model is compared with actual Bauschinger curves for metals in which the cell structure forms at 
room temperature. Our purposes are to examine whether or when the present model can explain actual Bauschinger curves and to search for a way to normalize them. A unified representation of the Bauschinger curves, if it is possible, will be interesting from the viewpoint of practical metal-forming.

A detailed consideration on the formation of cell structure and on the dislocation rearrangement in the structure during recovery and reversed loading will be presented in a unified form elsewhere ${ }^{(11)}$.

\section{The Model}

It may be reasonably assumed ${ }^{(12)}$ that point obstacles (forest dislocations in a pure metal) are distributed randomly on a slip plane and that the local resistance to dislocation glide varies from place to place in a non-uniform fashion, depending on the spacing of obstacles. Figure 1 illustrates a general view of flow and strain hardening ${ }^{(13)(14)}$. The resistance is high in the "black" (cross-hatched) area, and intermediate in the "grey" (hatched) one. In the white area, dislocations can glide rather

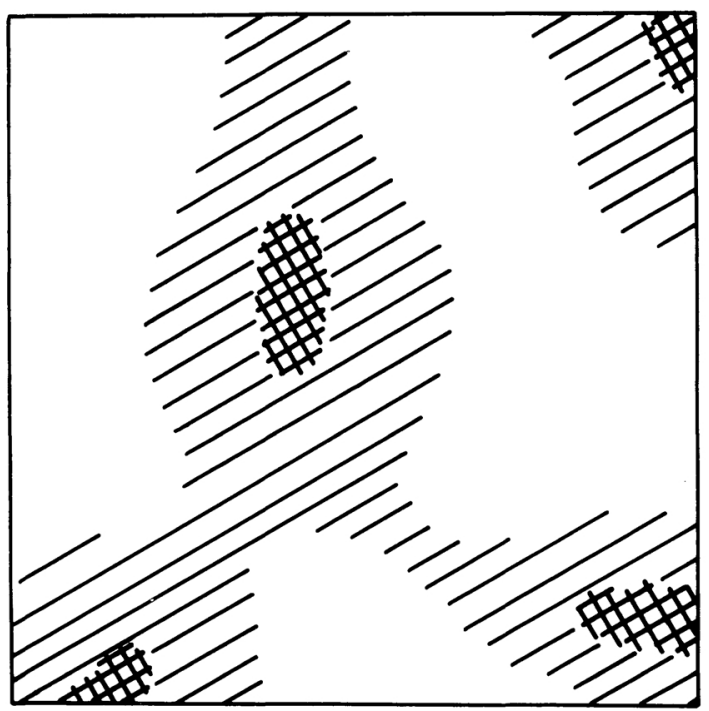

Fig. 1 Three kinds of area in a slip plane of a deformed metal, black (cross-hatched), grey (hatched) and white areas, correspond to the core of cell wall, the cell wall and the cell interior, respectively. The Bauschinger strain is caused by the relaxation of dynamically stored dislocations in white areas. freely.

When dislocation glide is areal, processes occurring during macroscopic flow can be summarized as follows. The flow stress is just high enough to permit slip throughout the grey area, but not throughout the black one. Orowan-type concave loops ${ }^{(13)}$ are left around the black area (that is, in the grey area), forming isolated tangles. With the progress of flow, more dislocations are accumulated in the grey area until finally the slip plane clogs by a linking-up of the tangles into cell walls. In the white area, some dynamic dislocation structure (in the simplest geometry, "dynamic pileups') may be built up under the flow stress, giving rise to a "stored strain" in it. Here, we think, the black, grey and white areas correspond to the core of cell wall, the cell wall, and the cell interior, respectively. Incidentally, pile-up-type internal stresses inside the concave-loop groups (that is, inside the cell wall) may be relaxed by the activation of other nearby forest dislocations, resulting in the extension of in-plane cell walls into the third dimension $^{(12)}$. Hence, dislocations accumulated in the cell wall may have been stabilized for unloading and reversed loading probably by the formation of attractive junctions with forest dislocations ${ }^{\dagger}$.

Some relaxation of the dynamic structure in the cell interior, most probably "unpiling" of dynamic pile-ups, may occur during unloading and reversed loading. Since dislocations in this area seem to have been stabilized to some extent (by the same mechanism as in the cell wall), the unpiling process may take plane gradually. We presume here that most of the dynamically stored strain in the cell interior can be released during unloading and reversed loading, causing the Bauschinger strain.

Assuming that the mean slip distance of dislocations approximately equals the cell diameter, $\lambda$, the stored strain, which essentially

$\dagger$ When the tensile prestress level has almost been reached in compression, a dissolution of cell walls was observed by TEM in aluminium ${ }^{(15)}$ and copper $^{(16)}$. A reduction of strain-hardening rate was associated with this phenomenon $^{(17)}$. However, the stress vs strain behaviour in such a later stage of reversed loading will not be discussed in the present paper. 
equals the Bauschinger strain, $\varepsilon_{\mathrm{r}}$, is given by eq. (1).

$$
\begin{aligned}
\varepsilon_{\mathrm{r}} & =b \lambda \rho_{\mathrm{d}} \\
& =b \lambda \rho \cdot \rho_{\mathrm{d}} / \rho
\end{aligned}
$$

where $b$ is the Burgers vector, $\rho_{\mathrm{d}}$ the density of dislocations dynamically stored in the cell interior and $\rho$ the total dislocation density. Combining the following relations (2), (3) and (4) with eq. (1),

$$
\begin{aligned}
\sigma_{\mathrm{p}} & =\sigma_{0}+M \alpha \mu b \sqrt{\rho} \\
\lambda & \simeq K l \\
l & \equiv 1 / \sqrt{\rho}
\end{aligned}
$$

( $\rho_{\mathrm{p}}$ is the prestress, $\sigma_{0}$ the friction stress which is negligibly small in a pure metal, $M$ the Taylor factor $(\simeq 3), \alpha$ a constant $(\simeq 1)^{(18)}, \mu$ the shear modulus, $l$ the average obstacle spacing and $K$ a constant $(\simeq 10)^{(12)}$, one can get eq. (5) for the Bauschinger strain, $\varepsilon_{\mathrm{r}}$ :

$$
\varepsilon_{\mathrm{r}}=K / M \alpha \cdot \sigma_{\mathrm{p}} / \mu \cdot f_{\mathrm{d}},
$$

where $f_{\mathrm{d}}=\rho_{\mathrm{d}} / \rho$, the fraction of reversely mobile dislocations. Thus, the Bauschinger strain, $\varepsilon_{\mathrm{r}}$, should be proportional to the prestress, $\sigma_{\mathrm{p}}$.

The applied stress, $\sigma$, at any stage should be scaled by the flow stress also (i.e., the prestress, $\left.\sigma_{\mathrm{p}}\right)^{(19)}$. Therefore, one can expect that the Bauschinger curves should become coincident after the normalization using $\sigma_{\mathrm{p}}$; that is, similarity in shape should hold between $\sigma / \sigma_{\mathrm{p}}$ vs $\varepsilon_{\mathrm{r}} / \sigma_{\mathrm{p}}$ curves. Incidentally, the factor $f_{\mathrm{d}}$ might vary with dislocation structure, probably being small for the well-defined subgrain structure and large for the planar arrays of dislocations. Hence, fears may be entertained that the normalization will not be applicable to a change in the nature of dislocation structure.

Woolley $^{(4)}$ originally showed the similarity among $\sigma / \sigma_{\mathrm{p}}$ vs $\varepsilon_{\mathrm{r}} / \sigma_{\mathrm{p}}$ curves in roomtemperature torsion tests of various fcc metals without mentioning any clear reason for the normalization by $\sigma_{\mathrm{p}}$. The present model may give a good reason for his treatment. Further, Ono et al. ${ }^{(20)}$ presented a sophisticated model for the Bauschinger effect by assuming the existence of a range of internal stresses in a prestrained metal and proposed a way of nor- malization by the amount of strain hardening, $\Delta \sigma$, though actual dislocation structures were not taken into account. The difference in the normalization parameter $\left(\sigma_{\mathrm{p}}\right.$ or $\left.\Delta \sigma\right)$ arises from the point whether one can ignore $\sigma_{0}$ (see eq. (2)) or not, and the present authors agree that $\Delta \sigma$ may be more suitable than $\sigma_{\mathrm{p}}$ for theoretical approach to the effect. In aluminium and copper examined in the present work, however, the degree of similarity among normalized Bauschinger curves was approximately equal with both parameters, and the same conclusions were drawn. Further, the use of $\sigma_{\mathrm{p}}$ may be more convenient for practical engineering. Therefore, in $\S \mathrm{IV}$, Bauschinger curves normalized by $\sigma_{\mathrm{p}}$ will be presented.

\section{Experimental Procedure}

Specimens for mechanical tests with a gauge length of $8 \mathrm{~mm}$ and a cross section of $5 \times 5$ $\mathrm{mm}^{2}$ were machined from cross-rolled sheets of polycrystalline aluminium (purity: 99.9 mass\%) and copper (99.99 mass\%) in which cell structure forms during room-temperature deformation. They were annealed in vacuum $\left(10^{-3} \mathrm{~Pa}\right)$ at several temperatures for $3.6 \mathrm{ks}$. The grain size after recrystallization was in the range from 70 to $440 \mu \mathrm{m}$. No evidence of recrystallization texture was detected by $\mathrm{X}$; ray Laue examination. The contents of main impurities by mass \% were as follows: in aluminium, Si 0.05, Fe 0.05, Cu 0.003, Mn $0.001, \mathrm{Mg}<0.001$, Ni 0.001 and $\mathrm{Cr}<0.001$; in copper, $\mathrm{O}<0.0003$ and others 0.0053 (in total).

Tension - compression tests (compression tests after tensile prestraining) were done at temperatures between 293 and $773 \mathrm{~K}$ under nominal strain rates of the order of $10^{-4} \mathrm{~s}^{-1}$ with an Instron-type machine modified for tension-compression cyclic loading. A detailed description of the machine has been given in a previous paper ${ }^{(7)}$.

\section{Experimental Results and Discussion}

\section{Effect of prestrain}

Figure 2 illustrates typical true stress-true 


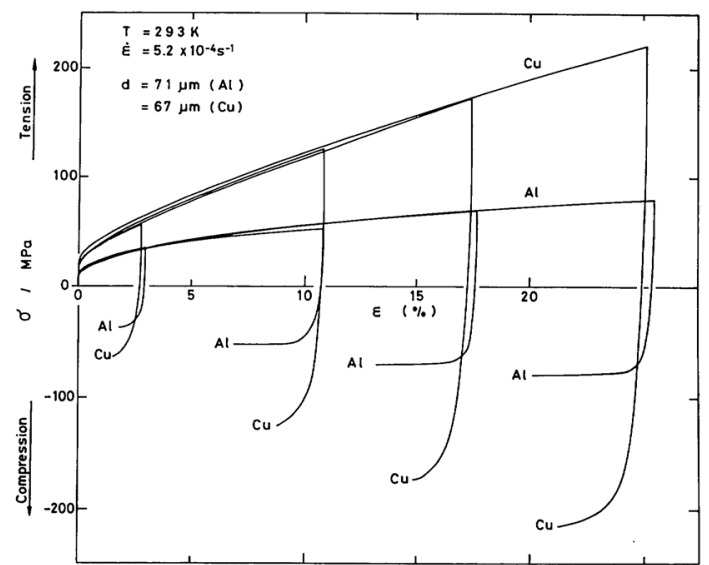

Fig. 2 True stress, $\sigma$, vs true strain, $\varepsilon$, curves of aluminium and copper at room temperature $(T=293 \mathrm{~K})$; grain size $d=\sim 70 \mu \mathrm{m}$ and strain rate $\dot{\varepsilon}=5.2 \times 10^{-4} \mathrm{~s}^{-1}$.

strain curves at $293 \mathrm{~K}$. In both metals, several phenomena such as the drop of yield stress, the appearance of Bauschinger strain and the "permanent softening" are seen during unloading and reversed loading, indicating the occurrence of the Bauschinger effect. Further, a strain region where the flow stress remains nearly constant ${ }^{(21)}$ is seen in aluminium, the origin of which has been discussed previously ${ }^{(17)}$.

As a first step, only the stress in the reversed direction, $\sigma$, was tentatively normalized by the prestress, $\sigma_{\mathrm{p}}$. Figure 3 shows $\sigma / \sigma_{\mathrm{p}}$ vs $\varepsilon_{\mathrm{r}}$ curves after stress reversal. As seen in the figure, the curves are not similar to one another at all in shape; $\varepsilon_{\mathrm{r}}$ at a given $\sigma / \sigma_{\mathrm{p}}$ increases with increasing prestrain, $\varepsilon_{\mathrm{p}}$.

The same data are presented in the form of $\sigma / \sigma_{\mathrm{p}}$ vs $\varepsilon_{\mathrm{r}} / \sigma_{\mathrm{p}}$ curves in Fig. 4 . The curves are virtually coincident, though they show a slight tendency to shift to the left with increasing $\varepsilon_{\mathrm{p}}$ for $\varepsilon_{\mathrm{p}}<\sim 3 \%$. According to TEM observation, the dislocation distribution was rather uniform for $\varepsilon_{\mathrm{p}}<\sim 3 \%$ in most of grains examined, but a cell structure had certainly formed at $\varepsilon_{\mathrm{p}}=\sim 7 \%$.

It may be concluded from results mentioned above that there exist two regions in prestraining: A transient region $\left(\varepsilon_{\mathrm{p}}<\sim 3 \%\right)$ and a quasi-steady region $\left(\varepsilon_{\mathrm{p}}>\sim 7 \%\right)^{\dagger \dagger}$. The former corresponds to a strain region where the cell structure has not yet formed, and the Bauschinger curves after prestraining into this region can not be uniquely represented by the

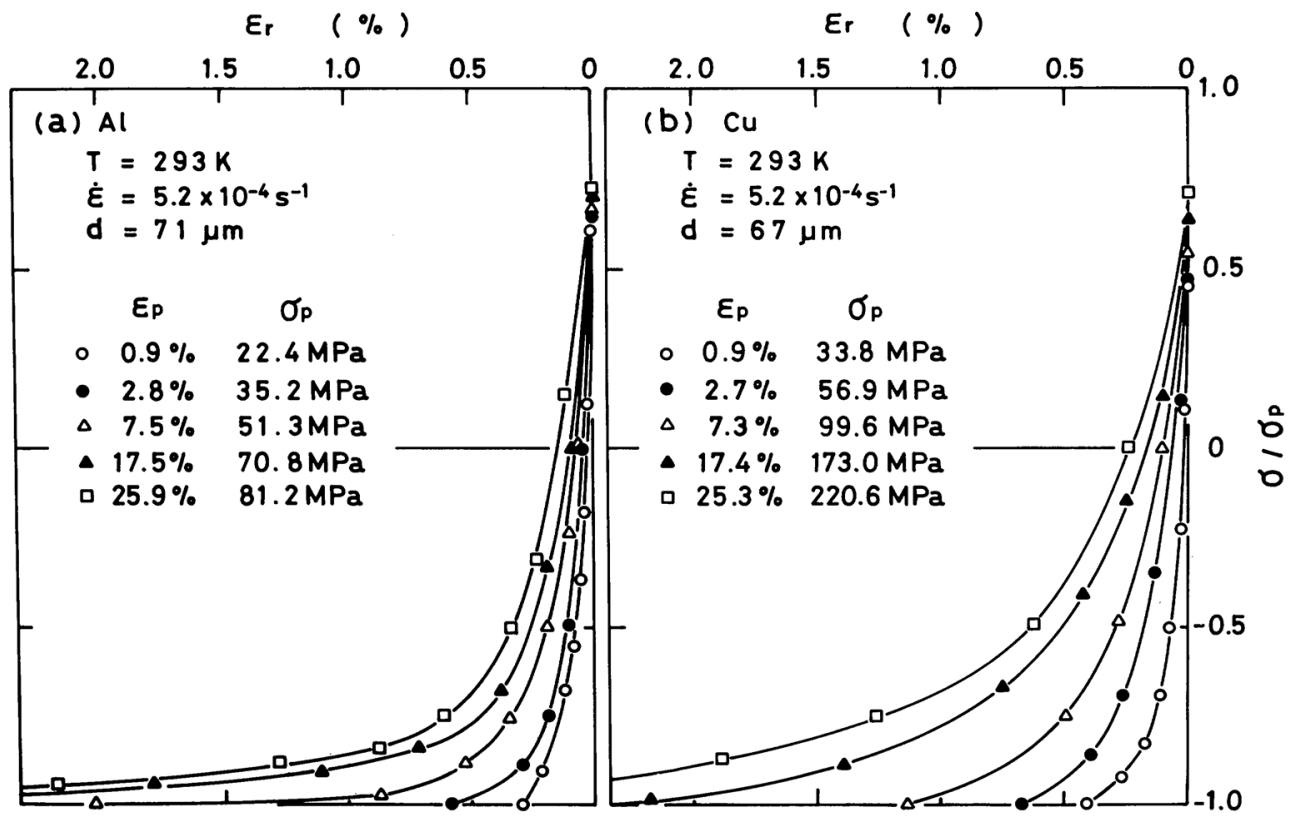

Fig. $3 \sigma / \sigma_{\mathrm{p}}$ vs $\varepsilon_{\mathrm{r}}$ curves in reversed direction at room temperature $(T=293 \mathrm{~K})$ ( $\sigma$ : true stress during unloading and reversed loading, $\varepsilon_{\mathrm{r}}$ : true strain in reversed direction, $\sigma_{\mathrm{p}}$ : prestress, $\varepsilon_{\mathrm{p}}$ : prestrain); grain size $d=\sim 70 \mu \mathrm{m}$ and strain rate $\dot{\varepsilon}=5.2 \times 10^{-4} \mathrm{~s}^{-1}$. (a) Aluminium and (b) copper. 


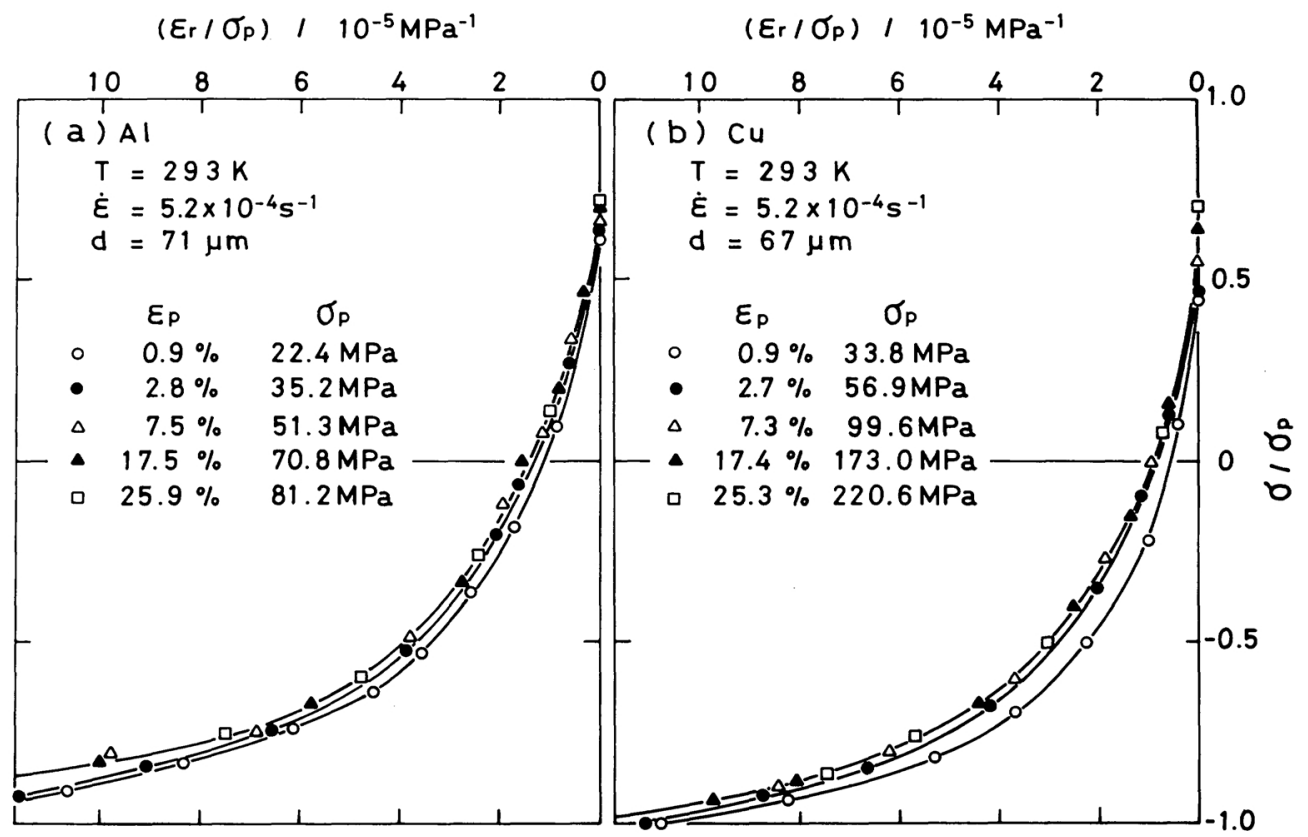

Fig. 4 Effect of prestrain, $\varepsilon_{\mathrm{p}}$, on $\sigma / \sigma_{\mathrm{p}}$ vs $\varepsilon_{\mathrm{r}} / \sigma_{\mathrm{p}}$ curves at room temperature $(T=293 \mathrm{~K}$ ) (on symbols, see Fig. 3). (a) Aluminium and (b) copper.

normalization using $\sigma_{\mathrm{p}}$. In the latter region where the cell structure has already been developed, on the other hand, the normalized Bauschinger curves become similar in shape, indicating the validity of the present model. The existence of the transient region can easily be envisaged from the fact that, in principle, the Bauschinger effect does not appear without strain hardening, namely at very small $\varepsilon_{\mathrm{p}}$. Further, the shift of curves to the left in this region seems to arise from an increase in $f_{\mathrm{d}}$ (in eq. (5)) due to an increase in the number of isolated dislocations, as for a decrease in the stackingfault energy (see §IV. 4).

\section{Effect of grain size}

Normalized $\sigma / \sigma_{\mathrm{p}}$ vs $\varepsilon_{\mathrm{r}} / \sigma_{\mathrm{p}}$ curves for dif-

It One might argue that the prestress is more appropriate than the prestrain for characterizing the dislocation structure and hence these two regions. Then, one may be able to say that the boundary-value of prestress between the regions $\left(\sigma_{\mathrm{p}}=35-50 \mathrm{MPa}\right.$ in aluminium and 55-100 MPa in copper, see Fig. 4) roughly corresponds to the (shear) stress $\tau_{\text {III }}$ for the beginning of stage III in single crystals (for similar purity to the present materials, $\tau_{\mathrm{III}}=\sim 13 \mathrm{MPa}$ in aluminium ${ }^{(22)}$ and $20-35$ $\mathrm{MPa}$ in copper ${ }^{(23)}$ ), because $\sigma_{\mathrm{p}} \simeq M \tau_{\mathrm{III}}$. ferent grain sizes, $d$, in the transient region $\left(\varepsilon_{\mathrm{p}}=2.8 \%\right)$ are shown in Fig. 5. The curves slightly tend to shift to the left with a decrease in $d$. This tendency is a little more remarkable in copper than in aluminium.

However, as seen in Fig. 6, even this small grain-size effect apparently disappears after prestraining into the quasi-steady region $\left(\varepsilon_{\mathrm{p}}=17.5 \%\right)$, again suggesting the validity of the present model after cell formation.

Dotted curves in Fig. 5 are the normalized Bauschinger curves after prestraining into the quasi-steady region, i.e. those given in Fig. 6 . It may be seen from the relative positions of the curves that the quasi-steady region is reached at smaller $\varepsilon_{\mathrm{p}}$ for smaller $d$. This was explained on the basis of the previous TEM observation that cell structures developed first in the vicinity of grain boundaries ${ }^{(24)}$.

\section{Effect of deformation temperature}

Figure 7 shows the effect of deformation temperature on $\sigma / \sigma_{\mathrm{p}}$ vs $\varepsilon_{\mathrm{r}} \mu / \sigma_{\mathrm{p}}$ curves after prestraining to $\varepsilon_{\mathrm{p}}=8-9 \%$. Since the shear modulus, $\mu$, changed with temperature, $\varepsilon_{\mathrm{r}}$ was normalized by $\sigma_{\mathrm{p}} / \mu$ (instead of $\sigma_{\mathrm{p}}$ ) according 
$\left(\varepsilon_{r} / \sigma_{p}\right) / 10^{-5} \mathrm{MPa}^{-1}$

$$
\left(\varepsilon_{\mathrm{r}} / \sigma_{\mathrm{p}}\right) / 10^{-5} \mathrm{MPa}^{-1}
$$

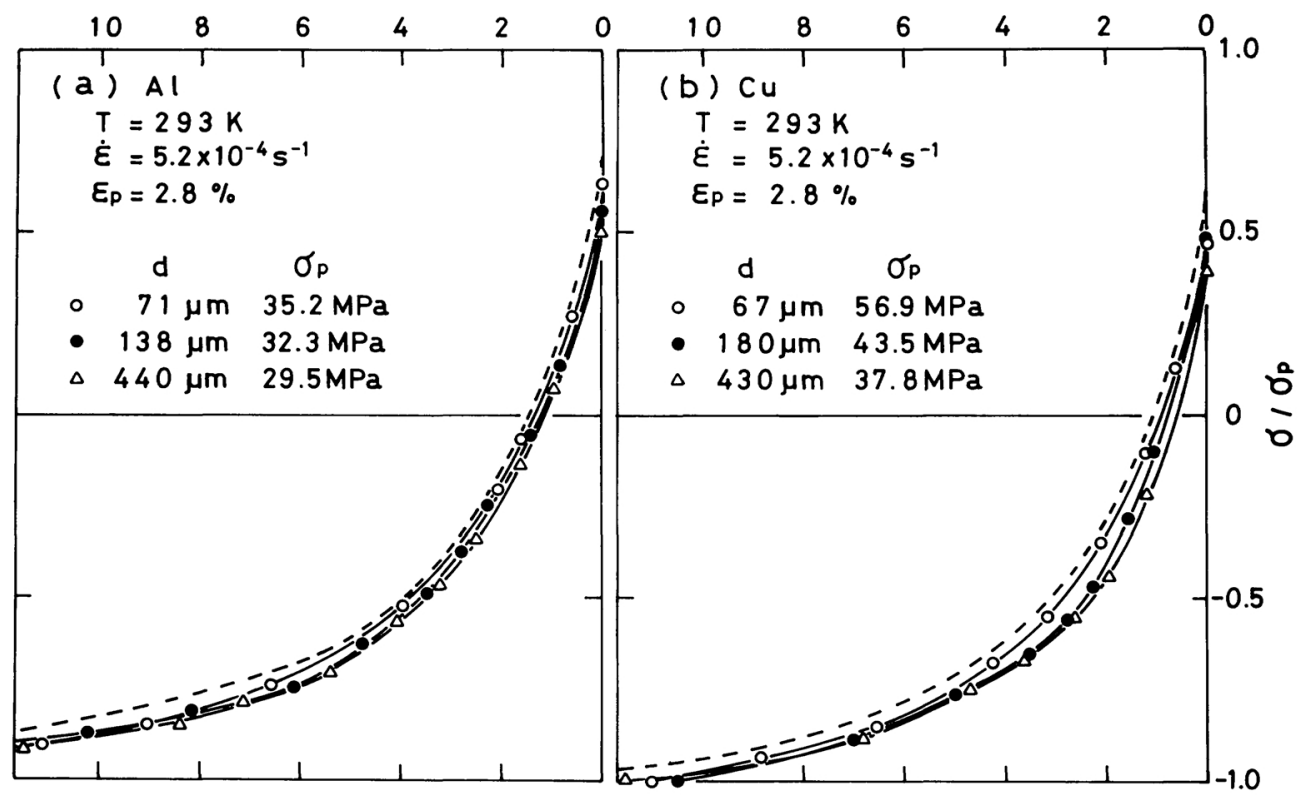

Fig. 5 Effect of grain size, $d$, on $\sigma / \sigma_{\mathrm{p}}$ vs $\varepsilon_{\mathrm{r}} / \sigma_{\mathrm{p}}$ curves at room temperature $(T=293 \mathrm{~K})$ after prestraining into transient region (on symbols, see Fig. 3). (a) Aluminium and (b) copper.

$\left(\varepsilon_{r} / \sigma_{p}\right) / 10^{-5} \mathrm{MPa}^{-1}$

$\left(\varepsilon_{r} / \sigma_{p}\right) / 10^{-5} \mathrm{MPa}^{-1}$

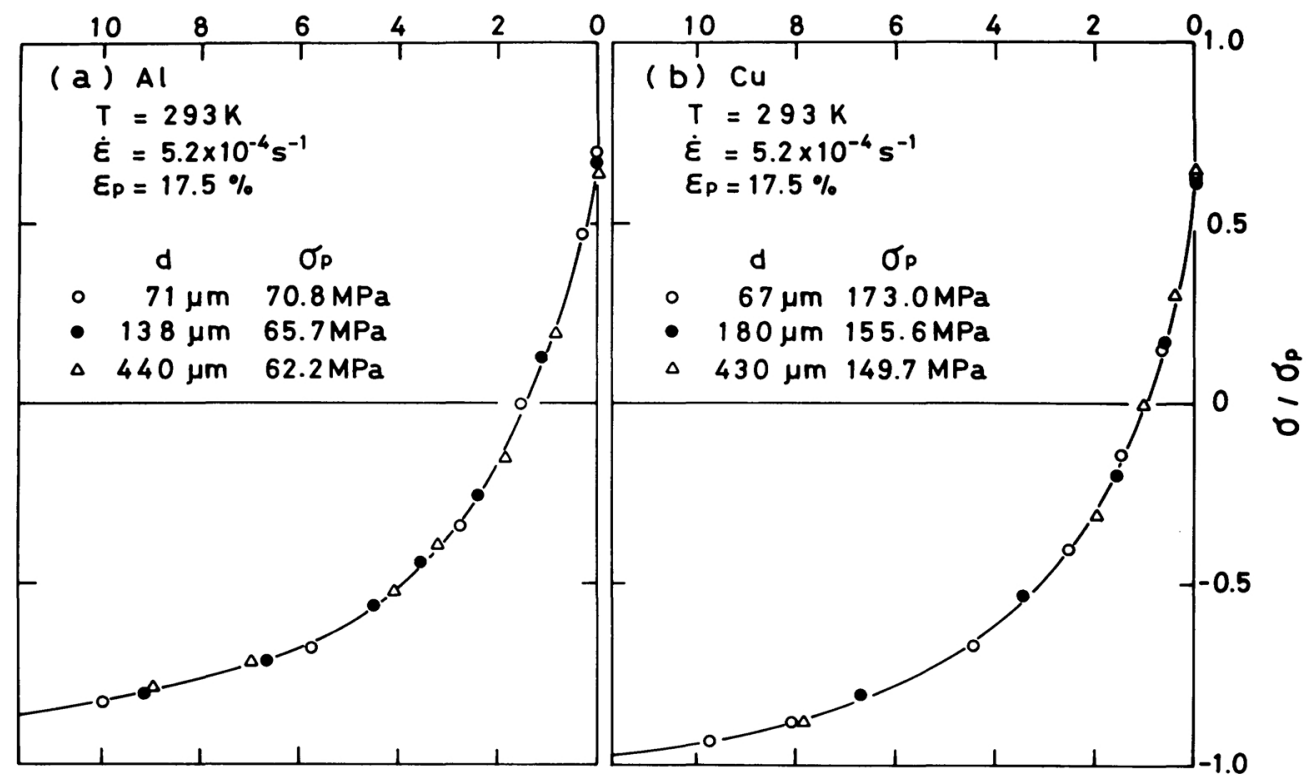

Fig. 6 Effect of grain size, $d$, on $\sigma / \sigma_{\mathrm{p}}$ vs $\varepsilon_{\mathrm{r}} / \sigma_{\mathrm{p}}$ curves at room temperature $(T=293 \mathrm{~K})$ after prestraining into quasi-steady region (on symbols, see Fig. 3). (a) Aluminium and (b) copper. 


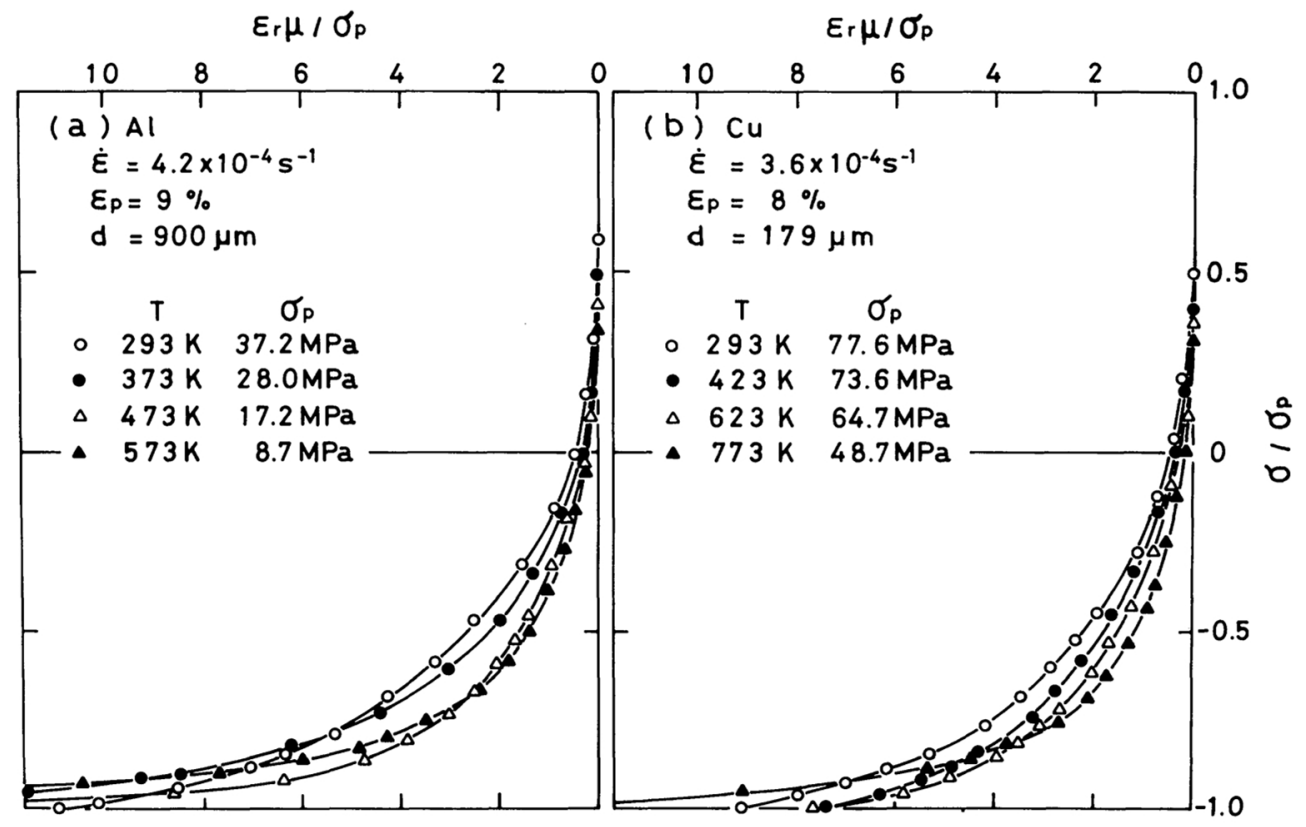

Fig. 7 Effect of deformation temperature, $T$, on $\sigma / \sigma_{\mathrm{p}}$ vs $\varepsilon_{\mathrm{r}} \mu / \sigma_{\mathrm{p}}$ curves after prestraining into quasi-steady region ( $\mu$ : shear modulus at $T$; on symbols, see Fig. 3). (a) Aluminium and (b) copper.

to eq. (5). Values of $\mu$ at deformation temperatures were estimated from those of the Young's modulus ${ }^{(25)}, E$, and the Poisson's ratio $^{(26)}, v$, using an equation $\mu=E / 2(1+v)$. Taking into account that the strain required for cell formation decreases with increasing temperature ${ }^{(27)}$, the amount of $\varepsilon_{\mathrm{p}}(8-9 \%)$ in the present case may be enough for prestraining to enter the quasi-steady region.

It is clear from the figure that the curves shift markedly to the right with increasing temperature, the normalization based on the present model being not applicable to the temperature change.

It is well known that, with increasing deformation temperature, the dislocation configuration changes from a cell structure to subgrain structure, accompanied by a decrease of dislocation density in the interior of cells or subgrains at a given $\operatorname{strain}^{(28)(29)}$. The most plausible reason for the unapplicability of the present model may be this conversion of dislocation structure. That is, because of faster recovery at higher temperatures, the value of $f_{\mathrm{d}}$ in eq. (5) may decrease with increasing temperature, causing a shift of the curves to
Table 1 Estimated values of $f_{\mathrm{d}}\left(=\rho_{\mathrm{d}} / \rho ; \rho_{\mathrm{d}}\right.$ : density of dislocations dynamically stored in the interior of cells or subgrains, $\rho$ : total dislocation density) in aluminium and copper.

$$
\mathrm{Al}, \varepsilon_{\mathrm{p}}=9 \%
$$

\begin{tabular}{ccc}
\hline \hline Temp. (K) & $f_{\mathrm{d}}$ at $\sigma / \sigma_{\mathrm{p}}=0$ & $f_{\mathrm{d}}$ at $\sigma / \sigma_{\mathrm{p}}=-0.5$ \\
\hline 293 & 0.063 & 0.371 \\
373 & 0.048 & 0.300 \\
473 & 0.038 & 0.240 \\
573 & 0.032 & 0.231 \\
\hline \hline \multicolumn{3}{c}{} \\
\hline \hline Temp. (K) & $f_{\mathrm{d}}$ at $\sigma / \sigma_{\mathrm{p}}=0$ & $f_{\mathrm{d}}$ at $\sigma / \sigma_{\mathrm{p}}=-0.5$ \\
\hline 293 & 0.063 & 0.300 \\
423 & 0.047 & 0.243 \\
623 & 0.035 & 0.218 \\
773 & 0.026 & 0.179 \\
\hline \hline
\end{tabular}

the right.

By definition $f_{\mathrm{d}}$ should be smaller than unity. Values of $f_{\mathrm{d}}$ obtained in the quasi-steady region are given in Table 1 . They decrease indeed with increasing temperature in both metals. Although we do not know at present which value $f_{\mathrm{d}}$ should take, estimated values of 
$\sim 0.4$ to $\sim 0.03$ seem, nevertheless, to be realistic.

\section{Comparison between metals examined}

Together with the deformation temperature, the stacking-fault energy influences the dislocation configuration developed during deformation. Figure 8 illustrates $\sigma / \sigma_{\mathrm{p}}$ vs $\varepsilon_{\mathrm{r}} \mu / \sigma_{\mathrm{p}}$ curves at $293 \mathrm{~K}$ after prestraining into the quasisteady region $\left(\varepsilon_{\mathrm{p}}=17.5 \%\right)$. Here, $\alpha$-brass (in mass \%, Cu 69.2, Zn 30.7; main impurities: $\mathrm{Fe}$ $0.004, \mathrm{~Pb} 0.001$ ) was also examined in addition to aluminium and copper. In $\alpha$-brass, only the planar arrays of dislocations were observed by TEM even at large prestrains.

As seen in the figure, curves shift to the left in the order of aluminium, copper and $\alpha$ brass, namely as the stacking-fault energy, $\gamma$, decreases $\left(\gamma=\sim 200, \sim 55\right.$ and $\sim 13 \mathrm{~mJ} / \mathrm{m}^{2}$ for aluminium, copper and $\alpha$-brass, respectively $\left.{ }^{(30)}\right)$. This implies that the present model can not be used for normalizing the variation of Bauschinger curves with the stacking-fault energy.

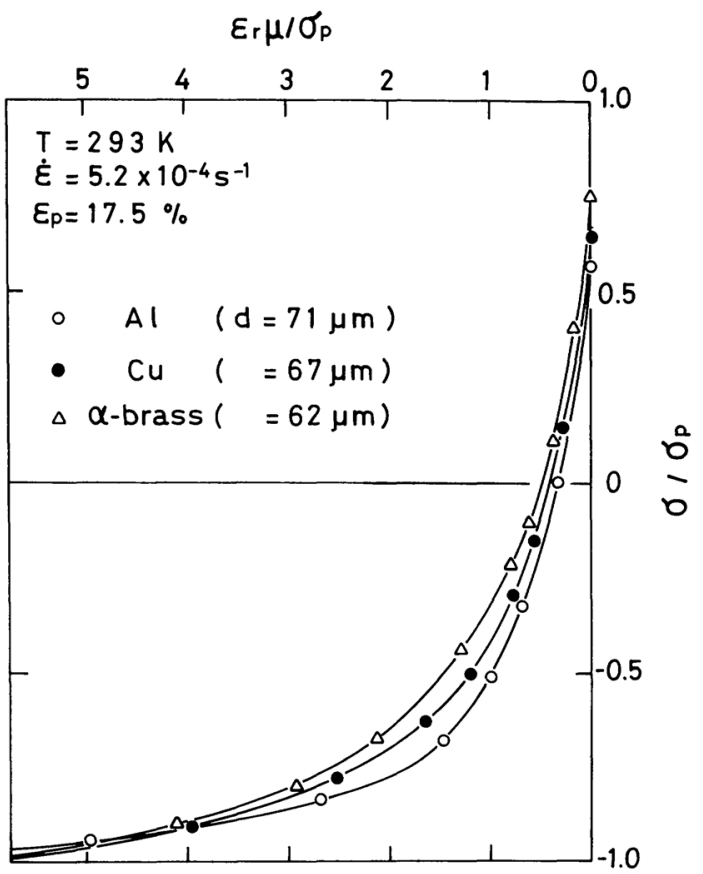

Fig. $8 \sigma / \sigma_{\mathrm{p}}$ vs $\varepsilon_{\mathrm{r}} \mu / \sigma_{\mathrm{p}}$ curves at room temperature $(T=293 \mathrm{~K})$ for aluminium, copper and $\alpha$-brass after prestraining into quasi-steady region ( $\mu$ : shear modulus; on symbols, see Fig. 3).
It is well known that the development of cell structure is less pronounced in metals with lower stacking-fault energies, resulting in the so-called planar structure with a high density of isolated dislocations ${ }^{(31)}$. Hence, the shift of normalized curves to the left in Fig. 8 may be ascribed to an increase in the density of dynamically stored dislocations which have not been associated with tangles and/or cell walls, namely to an increase in $f_{\mathrm{d}}$ (eq. (5)).

\section{Summary}

A model of the Bauschinger effect for cellforming metals was summarized, in which the Bauschinger strain was caused by "unpiling" of dynamic dislocation pile-ups in the cell interior developed during prestraining. To examine the validity of the model, it was compared with actual Bauschinger curves obtained in aluminium and copper (also in $\alpha$-brass in some cases). Main items of the conclusion drawn from the present work are summarized as follows:

(1) It is expected from the model that, when both stress, $\sigma$, and strain, $\varepsilon_{\mathrm{r}}$, in the reversed direction are normalized by the prestress, $\sigma_{\mathrm{p}}, \sigma / \sigma_{\mathrm{p}}$ vs $\varepsilon_{\mathrm{r}} / \sigma_{\mathrm{p}}$ curves become coincident with one another.

(2) In room-temperature deformation, prestraining falls into two regions, depending on an amount of prestrain, $\varepsilon_{\mathrm{p}}$ : a transient region $\left(\varepsilon_{\mathrm{p}}<\sim 3 \%\right)$ and a quasi-steady region $\left(\varepsilon_{\mathrm{p}}>\sim 7 \%\right)$. The boundary-value of prestrain between these two regions roughly corresponds to that required for cell formation. Although the normalization by $\sigma_{\mathrm{p}}$ was not applicable in the transient region, $\sigma$ vs $\varepsilon_{\mathrm{r}}$ behaviour for different prestrains and grain sizes after prestraining into the quasi-steady region was able to be almost completely normalized using $\sigma_{\mathrm{p}} ; \sigma / \sigma_{\mathrm{p}}$ vs $\varepsilon_{\mathrm{r}} / \sigma_{\mathrm{p}}$ curves became similar in shape. This indicates that the present model is valid when a cell structure has been developed during prestraining.

(3) However, the normalization by $\sigma_{\mathrm{p}}$ was not applicable to the variation of deformation temperature or stacking-fault energy. This suggests that the present way of normalization can not be used when a change in the nature of 
dislocation structure (such as the conversion of cell structure to subgrain structure or to planar arrays) occurs. This unapplicability may arise from a change in $f_{\mathrm{d}}=\rho_{\mathrm{d}} / \rho\left(\rho_{\mathrm{d}}\right.$ and $\rho$ are the density of dynamically stored dislocations and the total dislocation density, respectively) in eq. (5); $f_{\mathrm{d}}$ tends to decrease with an increase in deformation temperature or stacking-fault energy.

\section{Acknowledgements}

This work was sponsored by the Japan Society for the Promotion of Science; we thank Prof. R. Horiuchi for arranging an invitation for U.F.K. to spend six weeks in Japan. This work was also supported by the Light Metals Educational Foundation of Japan.

\section{REFERENCES}

(1) J. Bauschinger: Zivilinginieur, 27 (1881), 289.

(2) A. H. Cottrell: Dislocations and Plastic Flow in Crystals, Oxford Univ. Press, (1953), p. 111.

(3) T. Kishi and T. Tanabe: J. Mech. Phys. Solid, 21 (1973), 303.

(4) R. L. Woolley: Phil Mag., 44 (1953), 597.

(5) S. N. Buckley and K. M. Entwistle: Acta Met., 4 (1956), 325.

(6) A. Abel and H. Muir: Phil. Mag., 26 (1972), 489.

(7) T. Hasegawa, T. Yakou, M. Shimizu and S. Karashima: Trans. JIM, 17 (1976), 414.

(8) T. Yakou, T. Hasegawa, E. Shimokawa and S. Karashima: Trans. JIM, 18 (1977), 25.

(9) A. Seeger: Kristallplastizität, Handbuch der Physik, Band VII/2, Springer-Verlag, (1958), p. 1.

(10) P. B. Hirsch: The Relation Between the Structure and Mechanical Properties of Metals, Her Majesty's Stationary Office, (1963), p. 40.

(11) T. Hasegawa, T. Yakou and U. F. Kocks: To be presented in the International Conf. on Low Energy Dislocation Structures, Aug. 10-14, 1986, Univ. of
Virginia (accepted for publication in Mater. Sci. Eng.).

(12) U. F. Kocks: Dislocations and Properties of Real Materials, The Institute of Metals (London), (1985), p. 125.

(13) U. F. Kocks: Phil. Mag., 13 (1966), 541.

(14) U. F. Kocks: Constitutive Equations in Plasticity, ed. by A. S. Argon, MIT Press, (1975), p. 81.

(15) T. Hasegawa, T. Yakou and S. Karashima: Mater. Sci. Eng., 20 (1975), 267.

(16) N. Christodoulou, O. T. Woo and S. R. MacEven: to be published in Acta Met.

(17) T. Yakou, T. Hasegawa and S. Karashima: Trans. JIM, 26 (1985), 88.

(18) H. Mecking and U. F. Kocks: Acta Met., 29 (1981), 1865.

(19) U. F. Kocks, A. S. Argon and M. F. Ashby: Prog. Mater. Sci., 19 (1975), 1.

(20) N. Ono, D. J. H. Corderoy and H. Muir: Strength of Metals and Alloys, ed. by R. C. Gifkins, Pergamon Press, (1982), p. 431.

(21) T. Hasegawa and T. Yakou: Scr. Met.: 8 (1974), 951.

(22) B. Jaoul and C. Crussard: C. R. Acad. Sci., 234 (1952), 700; cited in Étude de la Plasticité et Application aux Métaux, by B. Jaoul, Dunod, (1965).

(23) J. Diehl: Z. Metallk., 47 (1956), 411.

(24) N. Hansen and B. Ralph: Acta Met., 30 (1982), 411.

(25) W. Köster: Z. Metallk., 39 (1948), 1.

(26) G. W. C. Kaye and T. H. Laby: Tables of Physical and Some Mathematical Functions, 13th edition, Longmans, (1966), p. 33.

(27) A. S. Keh and S. Weissman: Electron Microscopy and Strength of Crystals, ed. by G. Thomas and J. Washburn, Interscience, (1963), p. 231.

(28) M. R. Staker and D. L. Holt: Acta Met., 20 (1972), 569.

(29) D. J. Michel, J. Moteff and A. J. Lovell: Acta Met., 21 (1973), 1269.

(30) P. C. J. Gallagher: Met. Trans., 1 (1970), 2429.

(31) P. R. Swann: Electron Microscopy and Strength of Crystals, ed. by G. Thomas and J. Washburn, Interscience, (1963), p. 131. 